\title{
Editorial
}

\section{Profiling foods and diets}

Profiling, in the broadest sense, can be defined as the extrapolation of information about something, based on known qualities ${ }^{(1)}$. In nutrient profiling, we are attempting to assess the healthfulness of foods based on their nutrient content. 'Healthfulness' as a concept may seem overly broad and hard to quantify, but assigning a score based on a food's nutrient profile offers a way to gain an immediate understanding of where a food falls on a continuum based on our understanding of the properties of the nutrients within it.

In this issue of Public Health Nutrition, Nicklas et al. ${ }^{(2)}$ spell out the challenges in using nutrient profiling to identify nutrient-dense foods - among them, what criteria to use to define nutrient density, whether scoring leads to the unintended consequence of labelling foods as 'good' or 'bad', and whether nutrient-dense foods can be considered apart from the diets in which they are consumed. In an accompanying, invited commentary, Scarborough and Raynor $^{(3)}$ respond with a different perspective on what nutrient profiling is and when it is most useful - specifically, in situations in which foods can be considered apart from their diets, for example as a basis for decisions related to regulation and policy. The issues raised in both commentaries give much to consider as we decide on how best to develop and use nutrient profiling methods and scores.

Profiling need not stop at the food level; entire diets can be profiled as well. To complement the commentaries on nutrient profiling, this issue also includes several articles that demonstrate a variety of approaches to profiling overall diets and how such approaches can be used. Data from the US National Health and Nutrition Examination Survey are used by Powell-Wiley et al. to compute an index score based on nine target nutrients to quantify adherence to a Dietary Approaches to Stop Hypertension (DASH) dietary pattern $^{(4)}$. Roswall et al. define a 'Nordic' diet based on seven healthy food items and examine the consumption of these foods across ten countries in Europe ${ }^{(5)}$. Pot et al. describe their development of a simple, four-item Eating Choices Index based on breakfast consumption, fruit consumption, type of milk consumed and type of bread consumed, and find it to be moderately correlated with several nutrients of interest ${ }^{(6)}$.

Dietary diversity is another way to profile overall diets. In an investigation conducted in Kenya, Gewa et al. compare different ways to incorporate minimum intakes of nine different food groups into Diet Diversity Scores; their work offers a fascinating look at developing an approach to quantify diet quality in low-income countries ${ }^{(7)}$. Finally, in the only study that quantifies patterns empirically rather than based on an a priori selection of 'healthful' nutrients,
Harrington et al. use latent class analysis to identify three dietary patterns ('Western', 'healthy', low-energy') in the Cork and Kerry Diabetes and Heart Disease Study in Ireland and describe transitions in intake of these over time ${ }^{(8)}$.

Examples in this issue demonstrate a range of different approaches to understanding diet in its entirety and to quantifying its overall quality. Improving overall diet quality in order to improve health is, after all, the primary aim. While nutrient profiling of foods provides a tool to navigate the unprecedented variety of foods available in today's marketplace, this by itself seems a relatively short-term goal. The main question is this: How can nutrient profiling most effectively be used to encourage selection of foods that will lead to a measurably better-quality diet overall?

Marilyn Tseng

Editor-in-Chief

Email: mtseng@calpoly.edu

Allison Hodge

Deputy Editor

Email: allison.hodge@cancervic.org.au

\section{References}

1. Wikipedia (2013) Profiling http://en.wikipedia.org/wiki/ Profiling (accessed September 2014).

2. Nicklas TA, Drewnowski A \& O'Neil CE (2014) The nutrient density approach to healthy eating: challenges and opportunities. Public Health Nutr 17, 2626-2636.

3. Scarborough P \& Rayner M (2014) When nutrient profiling can (and cannot) be useful. Public Health Nutr 17, 2637-2640.

4. Powell-Wiley TM, Miller PE, Agyemang P et al. (2014) Perceived and objective diet quality in US adults: a cross-sectional analysis of the National Health and Nutrition Examination Survey (NHANES). Public Health Nutr 17, 2641-2649.

5. Roswall N, Olsen A, Boll K et al. (2014) Consumption of predefined 'Nordic' dietary items in ten European countries an investigation in the European Prospective Investigation into Cancer and Nutrition (EPIC) cohort. Public Health Nutr 17, 2650-2659.

6. Pot GK, Richards M, Prynne CJ et al. (2014) Development of the Eating Choices Index (ECI): a four-item index to measure healthiness of diet. Public Health Nutr 17, 2660-2666.

7. Gewa CA, Murphy SP, Weiss RE et al. (2014) Determining minimum food intake amounts for diet diversity scores to maximize associations with nutrient adequacy: an analysis of schoolchildren's diets in rural Kenya. Public Health Nutr 17, $2667-2673$

8. Harrington JM, Dahly DL, Fitzgerald AP et al. (2014) Capturing changes in dietary patterns among older adults: a latent class analysis of an ageing Irish cohort. Public Health Nutr 17, 2674-2686. 